\title{
Diffusion behavior of the benzene molecule in faujasite-type zeolite studied by double quantum filtered NMR $\dagger$
}

\author{
Yu-Huei Chen and Lian-Pin Hwang* \\ Department of Chemistry, National Taiwan University and Institute of Atomic and Molecular Sciences, Academia Sinica, Taipei, Taiwan
}

Received 4 February 1999; accepted 20 April 1999

\begin{abstract}
Deuteron NMR relaxation methods including double quantum filtered (DQF) spectral analyses were examined to study the dynamics of benzene molecules adsorbed in faujasite-type zeolite. To describe the dynamics of benzene molecules, a modified cone model involving in-plane rotation, wobbling motion and the characteristic order parameter was applied. The characteristic order parameter describes the intracage restricted reorientation for a single-site adsorption. For multiple-site adsorption such as in $\mathrm{NaY}$, the site-to-site hopping processes result in cage disorder and yield a small characteristic order parameter. The wobbling motion is induced by the diffusive motion and therefore the correlation time of wobbling motion may be invoked to estimate the self-diffusion coefficient of benzene adsorbed in NaY, DAY, USY and NaX. Comparisons with kinetic Monte Carlo calculation, pulsed field gradient measurement, and other NMR relaxation studies are discussed. Copyright (C) 1999 John Wiley \& Sons, Ltd.
\end{abstract}

KEYWORDS: NMR; ${ }^{2} \mathrm{H}$ NMR; benzene; diffusion; faujasite zeolite; relaxation; double-quantum

\section{INTRODUCTION}

Intracrystalline adsorption dynamics and cage-to-cage molecular diffusion have been studied extensively by several different experimental techniques. ${ }^{1-3}$ A detailed knowledge of intracrystalline diffusions of adsorbate in zeolite system is essential in understanding the catalytic and separation properties in these systems. The behavior of benzene in faujasite-type zeolite has been investigated both theoretically and experimentally in order to provide an understanding of the interactions and the molecular dynamics that take place between the zeolite framework and benzene molecules. ${ }^{4-7}$ A description of the theoretical backgroud of the NMR analysis of adsorption systems has been given by Pfeifer and co-workers. ${ }^{5}$ Recently, Schaefer et al. used two-dimensional (2D) exchange ${ }^{13} \mathrm{C}$ NMR techniques to study intracage hopping dynammics of benzene molecules strongly adsorbed on highly ordered Ca-LSX zeolite. ${ }^{8}$ Isfort et al. employed ${ }^{2} \mathrm{H} 2 \mathrm{D}$ NMR spectra and $2 \mathrm{D}$ echo time-domain decay profiles to obtain the relevant correlation function directly for the adsorption of benzene on NaY. ${ }^{9}$ Solid-state ${ }^{13} \mathrm{C}$ NMR exchange measurements of intercage hopping by strongly adsorbed benzene molecules in $\mathrm{Ca}-\mathrm{Y}$ give the motional correlation times and intracrystallite diffusion coefficients. ${ }^{10}$ However, experimental verifications of these prediction for benzene in

\footnotetext{
* Correspondence to: L.-P. Hwang, Department of Chemistry, National Taiwan University, Taipei, Taiwan.

E-mail: nmra@po.iams.sinica.edu.tw

$\dagger$ Dedicated to Professor Dr Dr Harry Pfeifer on the occasion of his 70th birthday.

Contract/grant sponsor: National Science Council of the Republic of China; Contract/grant number: NSC 88-2113-M-002-00.
}

most faujasite-type zeolites are not yet available because of the difficulty in measuring the self-diffusion coefficient for benzene in zeolite powder with small crystallites.

In the present series of deuteron NMR relaxation studies, double quantum filtered (DQF) spectral analysis was used to investigate the adsorption of perdeuterated benzene in USY, DAY and NaY. ${ }^{11,12}$ The validity of using DQF spectral analysis depends on the deuteron residual quadrupolar interaction, which in turn accounts for the non-vanishing interaction of benzene molecules on adsorption sites. By leaving out the overall reorientation of the whole molecule, the cone model of Brainard and Szabo is modified to facilitate the calculation of the timecorrelation function of the adsorbed benzene molecule. ${ }^{13}$ In this model, the wobbling motion with an order parameter is included to describe the restricted reorientational motion on adsorption sites. A characteristic order parameter is introduced to take into account the cage disorder due to site-to-site hopping. For a simple site adsorption such as in USY, since there is lack of site-to-site hopping, a larger characteristic order parameter may be expected in this system. For multiple-site adsorption such as in $\mathrm{NaY}$, the site-to-site hopping processes result in cage disorder and yield a small characteristic order parameter. ${ }^{12}$

A kinetic modeling study by the Monte Carlo method suggests that measuring benzene orientation randomization (BOR) in NaY can provide important information related to cage-to-cage molecular diffusion. ${ }^{4 e}$ In the present work, the application of the modified cone model to site-to-site hopping and wobbling motion processes is essentially similar to the BOR model used by Auerbach and Metiu. ${ }^{4 \mathrm{e}}$ In early part of the orientation correlation function, the orientation randomization in their model 
was found to be associated with the site-to-site hopping process. The long-time behavior of the orientational correlation function may be correlated with the $\tau_{\mathrm{w}}$ process in the present model since the wobbling motion is induced by the barriers of zeolite framework in the course of benzene diffusion through the cage. Furthermore, the long-time BOR rate is identified as the rate of cage-to-cage motions. In the light of these obvious connection between the longtime orientation randomization and the wobbling motion concerning cage-to-cage diffusion, it was the purpose of this work to establish an approach from more complete NMR relaxation studies to estimate the self-diffusion coefficient of benzene in faujasite-type zeolites. The results of DQF spectral analysis for benzene in $\mathrm{NaX}$ are discussed. A comparison with previous relaxation studies and pulsed field gradient (PFG) NMR measurements is also provided.

\section{FORMULATION OF THEORY}

\section{Two-site exchange model with residual quadrupolar interaction}

The adsorption interaction of perdeuterated benzene in zeolite may in turn be related to a residual quadrupolar interaction $\omega_{\mathrm{q}}$ of deuterium nuclei due to local order. The Hamiltonian of this interaction is given by ${ }^{14}$

$$
H=\omega_{\mathrm{q}, \theta}\left[I_{z}^{2}-I(I+1)\right]
$$

where $\omega_{\mathrm{q}, \theta} \equiv \omega_{\mathrm{q}}\left(3 \cos ^{2} \theta-1\right) / 2$ is the residual quadrupolar interaction in the laboratory frame ${ }^{15}$ and $I_{z}$ is the $z$ component of the spin operator with $I=1$ for deuterium nuclei. $\theta$ is the angle between the local symmetry axis of the quadrupolar interaction tensor and the Zeeman field. A model with two-site exchange between a fast motion site, denoted by $f$, and a slow motion site, denoted by $s$, is assumed in general. The $s$ (or $f$ ) site accounts for the adsorption (or non-adsorption) state of benzene in faujasite zeolites. An isotropic distribution of $\theta$ is applied to approximate the situation of the random orientational distribution of zeolite crystals in a powder sample.

DQF spectra are conventionally measured using the pulse sequence $\pi / 2-\tau-\pi / 2-\delta-\pi / 2-\mathrm{t}$ (acq.), where $\tau$ is the creation time for double quantum (DQ) coherence. The effect of this pulse sequence for spin-1 nuclei in a heterogeneous system can be found elsewhere. ${ }^{11,12}$ The evolution of related transverse components $\sigma_{11}$ and $\sigma_{21}$ at sites $f$ and $s$, expressed by the superscripts, is needed in the calculation of DQ coherence and the observable by following the rate equation

$$
\frac{\mathrm{d}}{\mathrm{d} t}\left[\begin{array}{c}
\sigma_{11}^{f} \\
\sigma_{21}^{f} \\
\sigma_{11}^{s} \\
\sigma_{21}^{s}
\end{array}\right]=\left[\begin{array}{cccc}
-R_{11}^{f}-k_{f s} & 0 & k_{s f} & 0 \\
0 & -R_{21}^{f}-k_{f s} & 0 & k_{s f} \\
k_{f s} & 0 & -R_{11}^{s}-k_{s f} & -i w_{\mathrm{q}, \theta} \\
0 & k_{f s} & -i w_{\mathrm{q}, \theta} & -R_{21}^{s}-k_{s f}
\end{array}\right]\left[\begin{array}{c}
\sigma_{11}^{f} \\
\sigma_{21}^{f} \\
\sigma_{11}^{s} \\
\sigma_{21}^{s}
\end{array}\right]
$$

where the residual quadrupolar interaction exists in $s$ sites only and $k_{f s}\left(k_{s f}\right)$ is the microscopic rate constant for transfer from site $f(s)$ to site $s(f)$. In addition, the detailed balancing of the exchange process demands that $P_{f} k_{f s}=P_{s} k_{s f}$, where we define $P_{f}$ and $P_{s}$ as the total populations in site $f$ and site $s$, respectively. In Eqn (2), the relaxation rate constants for single and double quantum coherences (expressed by the first subscript of $R)$ with different sites $\alpha(\alpha=s$ or $f)$ are given by $R_{11}^{\alpha}=C^{\alpha}\left(3 J_{0}^{\alpha}+5 J_{1}^{\alpha}+2 J_{2}^{\alpha}\right)$ and $R_{21}^{\alpha}=C^{\alpha}\left(3 J_{0}^{\alpha}+J_{1}^{\alpha}+\right.$ $\left.2 J_{2}^{\alpha}\right)$, where we have $C^{f} \equiv(3 / 160) \chi_{f}{ }^{2}$ and $C^{s} \equiv$ $(3 / 160) \chi_{s}^{2}$ and $\chi_{f}$ and $\chi_{s}$ are defined as the quadrupolar coupling constants $\chi_{f} / 2 \pi \equiv e^{2} Q q_{f} / h$ and $\chi_{s} / 2 \pi \equiv$ $e^{2} Q q_{s} / h$ for fast and slow motion sites, respectively. $J_{n}^{s}$ and $J_{n}^{f}$ are the spectral density functions, defined below for reorientational relaxation of benzene molecules in $f$ and $s$ sites, respectively. The contribution of the asymmetry parameter $(\eta=0.041)$ of the electric field gradient $e q$ to the coupling constant is neglected. ${ }^{16} J_{n}^{f}$ and $J_{n}^{s}$ are the spectral density functions defined below for reorientational relaxation of benzene molecules in $f$ and $s$ sites, respectively. Analogous simulations of the spectra have been described elsewhere. ${ }^{17}$ In the calculation of $T_{1}$, the time evolution of the longitudinal components follows the rate equation ${ }^{18}$

$$
\frac{\mathrm{d}}{\mathrm{d} t}\left[\begin{array}{c}
\sigma_{10}^{f} \\
\sigma_{10}^{s}
\end{array}\right]=\left[\begin{array}{cc}
-R_{10}^{f}-k_{f s} & k_{s f} \\
k_{f s} & -R_{10}^{s}-k_{s f}
\end{array}\right]\left[\begin{array}{c}
\sigma_{10}^{f} \\
\sigma_{10}^{s}
\end{array}\right]
$$

where $R_{10}^{f}$ and $R_{10}^{s}$ are the longitudinal relaxation rates for site $f$ and site $s$, respectively, as defined by $R_{10}^{\alpha}=$ $2 C^{\alpha}\left(J_{1}^{\alpha}+4 J_{2}^{\alpha}\right)$.

\section{Residual quadrupolar interaction in the adsorptive process}

The position with maximum $\omega_{\mathrm{q}}$, denoted by $\omega_{\mathrm{qm}}$, may be defined as the benzene molecule adsorbed in the adsorption site with maximum strength of interaction. Moreover, the motion associated with $\omega_{\mathrm{qm}}$ involves inplane motion around the hexad axis only and there is no wobbling motion of the ring normal. Thus, from both the experimental evidence and the symmetry arguments resulting from the fast internal motion around the hexad axis, the quadrupolar interaction of perdeuterated benzene molecules in this case reduces to half of the value obtained for rigid stationary benzene. ${ }^{19}$ Hence we take $\omega_{\mathrm{qm}}=1 / 2 \omega_{\mathrm{q} 0}$, where $\omega_{\mathrm{q} 0}=3 / 4 \chi$. We observe a powder pattern in the ${ }^{2} \mathrm{H}$ NMR spectrum for low-loading zeolite $\mathrm{NaY}$ at $130 \mathrm{~K} .^{12}$ The edge splitting $\Delta v$ of the $\mathrm{NaY}$ sample is $69 \mathrm{kHz}$, which implies a quadrupolar coupling constant $\chi / 2 \pi$ of $184 \mathrm{kHz}$. This result agrees with that of Pfeifer and co-workers ${ }^{5 a}$ and Gladder and co-workers ${ }^{7}$ and suggests that immobile benzene is undergoing only in-plane rotation. However, a solid pattern with a central peak appeared in ${ }^{2} \mathrm{H}$ NMR spectra for the USY sample measured at 135 and $150 \mathrm{~K} .{ }^{11}$ The appearance of a central peak may suggest that a slow exchange process still exists at these temperatures. From these two measurements the same $67 \mathrm{kHz}$ edge splittings in the USY sample are obtained. According to the above results, we may take an average value $\omega_{\mathrm{qm}}=68 \mathrm{kHz}$ or $\chi_{s} / 2 \pi=181 \mathrm{kHz}$ as the 
maximum value $\omega_{\mathrm{qm}}$ in our calculation for all of the faujasite samples. Moreover, the quadrupolar coupling constant at the fast site may be estimated for $\mathrm{C}_{6} \mathrm{D}_{6}$ in liquid crystal phase. $\chi_{f} / 2 \pi$ is estimated to be $183 \mathrm{kHz} .{ }^{16}$ Therefore, one may take $\chi_{f} / 2 \pi \approx \chi_{s} / 2 \pi=181 \mathrm{kHz}$ in our calculation.

\section{Spectral density function for benzene in fast motion sites}

In order to match the central peak widths of the DQF spectra, the motional narrowing conditions are required for $\tau_{f}$, which is defined as the tumbling correlation times of benzene in an $f$ site. Hence we have $J_{n}^{f} \approx 2 \tau_{f}$. The simulated results are insensitive to these two values as long as their magnitudes are kept smaller than $10^{-10} \mathrm{~s}$.

\section{Spectral density function for benzene in slow motion sites}

The spectral density functions for site $s$ with consideration of residual quadrupolar interaction are defined by ${ }^{18}$

$$
\begin{aligned}
J_{0}^{s} & \equiv j_{0}^{s}\left(1+\frac{10}{7}\left\langle D_{00}^{(2)}\right\rangle+\frac{18}{7}\left\langle D_{00}^{(4)}\right\rangle-5\left\langle D_{00}^{(2)}\right\rangle^{2}\right) \\
J_{1}^{s} & \equiv j_{1}^{s}\left(1+\frac{5}{7}\left\langle D_{00}^{(2)}\right\rangle-\frac{12}{7}\left\langle D_{00}^{(4)}\right\rangle\right) \\
J_{2}^{s} & \equiv j_{2}^{s}\left(1-\frac{10}{7}\left\langle D_{00}^{(2)}\right\rangle+\frac{3}{7}\left\langle D_{00}^{(4)}\right\rangle\right)
\end{aligned}
$$

where $D_{00}^{(n)}$ are the Wigner rotation matrix elements with the orientation relative to the laboratory frame, and \langle\rangle means taking the average over the coordinates of inplane and wobbling motions. For multiple adsorption sites, additional averaging over the motion of site-tosite hopping is performed. The cone model of Brainard and $\mathrm{Szabo}^{13}$ is invoked but with minor modification to consider the residual interaction and hence to facilitate the calculation of the spectral density functions $j_{n}^{s}$ of the adsorbed benzene molecule. It is given by ${ }^{13,20}$

$$
\begin{aligned}
j_{n}^{s} \equiv & 2\left\{\frac{3}{4} S^{2} \frac{4 D_{\mathrm{i}}}{\left(4 D_{\mathrm{i}}\right)^{2}\left(n \omega_{0}\right)^{2}}\right. \\
& +\frac{3}{4}\left(1-S^{2}\right) \frac{4 D_{\mathrm{i}}+2 D_{\mathrm{w}} /\left(1-S^{2}\right)}{\left[4 D_{\mathrm{i}}+2 D_{\mathrm{w}} /\left(1-S^{2}\right)\right]^{2}+\left(n \omega_{0}\right)^{2}} \\
& \left.+\frac{1}{4}\left(1-S^{2}\right) \frac{6 D_{\mathrm{w}} /\left(1-S^{2}\right)}{\left[6 D_{\mathrm{w}} /\left(1-S^{2}\right)\right]^{2}+\left(n \omega_{0}\right)^{2}}\right\}
\end{aligned}
$$

where $\omega_{0}$ is the Larmor frequency of deuterium nuclei. It is convenient to define the correlation times for the internal (i) rotation and the wobbling (w) motion as $\tau_{i} \equiv 1 / 4 D_{\mathrm{i}}$ and $\tau_{\mathrm{w}} \equiv 1 / 6 D_{\mathrm{w}}$, respectively. The wobbling motion is assumed to be within a cone with the director normal to the adsorption site. The distribution of the cone may be expressed by an order parameter $S$ given by $S \equiv$ $\left\langle D_{00}^{(2)}\right\rangle_{s}$, where the average over all the motions is defined in the frame $s$ of an adsorption site. With this model the second and third terms in Eqn (4) may be readily evaluated as $\left\langle D_{00}^{(2)}\right\rangle=S D_{00}^{(2)}(\theta)$ and $\left\langle D_{00}^{(4)}\right\rangle=\left\langle D_{00}^{(4)}\right\rangle_{s} D_{00}^{(4)}(\theta)$, where axial symmetry of the adsorption interaction and site distribution is assumed. To evaluate the $\left\langle D_{00}^{(4)}\right\rangle_{\mathrm{s}}$ term in terms of $S$, an isotropic distribution within a cone angle $\beta$ with the director is invoked. Brainard and Szabo obtained $S=\cos \beta(1+\cos \beta) / 2$. This yields $\left\langle D_{00}^{(4)}\right\rangle_{\mathrm{s}}=$ $(S / 8)\left[28 S-7(1+8 S)^{(1 / 2)}+1\right]$. Hence with this model, one may evaluate the spectral density functions for site $s$. Furthermore, it should be noted that $\tau_{\mathrm{i}}$ and $\tau_{\mathrm{w}}$ are used in an averaged sense, i.e. their magnitudes remain the same irrespective of the variation of $S$ along the adsorption coordinates at a given temperature.

\section{Order parameter and residual quadrupolar interaction}

Since both the $S$ and $\omega_{\mathrm{q}}$ parameters share the same degree of randomness after averaging by the same wobbling motion, one may relate $S$ to the residual quadrupolar anisotropy $\omega_{\mathrm{q}}$ by $S=\omega_{\mathrm{q}} / \omega_{\mathrm{qm}}$. Furthermore, the adsorbate distribution function may be expressed in terms of $S_{\mathrm{c}}$ with the definition $S_{\mathrm{c}}=\omega_{\mathrm{qc}} / \omega_{\mathrm{qm}}$ as the characteristic order parameter. It is noted that there is a difference in adsorbate population along the adsorption coordinate as a consequence of exchange and migration of the adsorbate among various sites in the supercage. Considering the jump diffusion and the trapping nature of benzene molecule on to the adsorption site in faujasite, a probability distribution may be represented suitably in an exponential form $f\left(\omega_{\mathrm{q}}\right)=N \exp \left(-\omega_{\mathrm{q}} / \omega_{\mathrm{qc}}\right)$, where $N$ is a normalization factor and $\omega_{\mathrm{qc}}$ characterizes a correlation frequency for resident sites in the $\omega_{\mathrm{q}}$ space. ${ }^{12}$ Beyond this value, the population of adsorbate molecules decreases significantly. It should be noted that all the calculated results are averaged over the adsorbate distribution. The pictorial representation of the exchange between $f$ and $s$ sites and the restricted motion along the adsorption coordinate may be found in a previous paper. ${ }^{12}$

For faujasite, with merely a single site in the supercage, the average over the adsorption coordinates with the help of $f\left(\omega_{\mathrm{q}}\right)$ represents the thermal kinetic origin of the adsorption process mentioned previously. Since $\omega_{\mathrm{q}}$ is the result after averaging over all the motions concerned, the effective $\omega_{\mathrm{q}}$ represents an average over site-to-site hopping for multiple-site adsorption. ${ }^{4}$ Therefore, with the site-to-site hopping process, one may expect a small effective residual quadrupolar interaction and thus a small $\omega_{\mathrm{c}}\left(\right.$ or $S_{\mathrm{c}}$ ) value in the system of symmetrically averaged adsorption sites. The tetrahedrally arranged $\mathrm{Na} S_{\text {II }}$ sites in the NaY system is the case studied in the present work.

\section{Estimation of the self-diffusion coefficient}

The estimation of self-diffusion coefficients may be made with the relation $D=\left\langle l^{2}\right\rangle / 6 \tau_{\mathrm{h}}$, where $\left\langle l^{2}\right\rangle$ is the average distance between the adsorption sites in two adjacent cage and $\tau_{\mathrm{h}}$ is the correlation time for intercage hopping. As an estimate, $l=11 \AA$, the distance between the centers 
of two supercages. In accordance with the kinetic Monte Carlo (KMC) calculation, ${ }^{4 \mathrm{e}}$ the long-term BOR rate, $k_{\mathrm{BOR}}$, in $\mathrm{NaY}$ is controlled by cage-to-cage motion. Considering the residence time on site to be much longer than the flight time of a hop, we have $k_{\mathrm{BOR}}=k_{\text {cage. }}$. In terms of the present model one obtains $k_{\mathrm{BOR}}=1 / \tau_{\mathrm{w}}$. Since $k_{\text {cage }}=1 / \tau_{\mathrm{h}}$, the self-diffusion coefficient of benzene may be calculated from

$$
D=\frac{\left\langle l^{2}\right\rangle}{6 \tau_{\mathrm{w}}}
$$

\section{EXPERIMENTAL}

The $\mathrm{NaX}(13 \mathrm{X}, \mathrm{Si}: \mathrm{A} 1=1.2)$ used was supplied and characterized by Union Carbide Danbury, CT, USA. The adsorbate material, $\mathrm{C}_{6} \mathrm{D}_{6}$, was purchased from Merck (Darmstadt, Germany). With a minimum purity of $99.5 \%$, no further purification process was used.

One small glass ball containing the required amount of $\mathrm{C}_{6} \mathrm{D}_{6}$ was sealed and then admitted to the upper part of the sample tube with glass-wool separating it from the zeolite in the lower part of the tube. The sample was dehydrated under a $10^{-3} \mathrm{~Pa}$ vacuum at $673 \mathrm{~K}$ for at least $12 \mathrm{~h}$. After cooling, the tube was sealed, leaving the dried zeolite and the $\mathrm{C}_{6} \mathrm{D}_{6}$-carrying ball in the tube. The $\mathrm{C}_{6} \mathrm{D}_{6}$-carrying ball was then broken by shaking the tube. The lower part of the sample tube was chilled in liquid $\mathrm{N}_{2}$. The section of the NMR tube containing the loaded zeolite powder was sealed and separated from the upper part containing the rest of the glass capillary ball. Subsequently, the sample was kept at $353 \mathrm{~K}$ for $12 \mathrm{~h}$ to ensure a homogeneous distribution of adsorbate on the zeolite. The reproducibility of relaxation data was checked to examine whether equilibrium was reached in all supercages over a period of 5 months. The preparation of high- and low-loading samples for $\mathrm{NaY}$ ( $\mathrm{Si}: \mathrm{A} 1=$ 2.4), DAY (Si : A1 = 15) and USY (Si: A1 = 40) has been described in previous papers. ${ }^{11,12}$ The loadings of $\mathrm{C}_{6} \mathrm{D}_{6}$ (molecules per supercage) in various samples are as follows: for low-loading samples NaY 0.5, DAY 0.8, USY 0.5, NaX 0.6 and for high-loading samples NaY 3.0, DAY 3.9, USY 3.2, NaX 3.8.

The ${ }^{2} \mathrm{H}$ NMR measurements were performed on Bruker MSL-500 and MSL-300 spectrometers operating at $\quad 76.78 \mathrm{MHz} \quad(11.75 \mathrm{~T})$ and $46.07 \mathrm{MHz} \quad(7.05 \mathrm{~T})$, respectively. For transverse relaxation studies, the linewidths $\Delta v_{1 / 2}$ were measured as the full width at halfheight from the single-pulse experiment. The magnetic inhomogeneity was estimated by comparing the Hahn spin-echo and linewidth measurement of liquid $\mathrm{C}_{6} \mathrm{D}_{6}$. The magnetic inhomogeneity was found to account for less than $5 \mathrm{~Hz}$ of the observed line width at half-height in the spectra. The longitudinal relaxation measurements were obtained using the inversion-recovery pulse sequence. The ${ }^{2} \mathrm{H} T_{1}$ and linewidth measurements were performed on Bruker MSL-500 and MSL-300 spectrometers operating at $76.78 \mathrm{MHz}(11.75 \mathrm{~T})$ and $46.07 \mathrm{MHz}(7.05 \mathrm{~T})$, respectively, with a $\pi / 2$ pulse length of about $15 \mu \mathrm{s}$. A delay of at least $5 T_{1}$ was allowed between scans for the $T_{1}$ and DQF spectra with proper phase cycling. DQF spectra were measured conventionally using the pulse sequence $\pi / 2-\tau-\pi / 2-\delta-\pi / 2-$ (acq.). ${ }^{11,12,21}$ Since there is no fielddependent effect, the DQF spectra were displayed at $76.78 \mathrm{MHz}$ only. The temperature was controlled to within $\pm 0.1^{\circ} \mathrm{C}$ and was calibrated using a methanol reference. There are errors of approximately $10 \%$ associated with $T_{1}$ and linewidth measurements, respectively.

\section{RESULTS}

Figures 1 and 2 show the experimental DQF spectra together with their corresponding simulation for low- and high-loading $\mathrm{NaX}$ samples, respectively, over a range of temperatures at $76.78 \mathrm{MHz}$. The parameters used in the simulation of DQF spectra, in fitting $T_{1}$ and in linewidth analysis are listed in Table 1 . The calculated and experimental $T_{1}$ values and linewidths are listed in Table 2 for comparison. Moreover, the details of simulation, fitting algorithm and the results for NaY, DAY and USY system may be found in previous papers. ${ }^{11,12}$ In Table 3, we only list the values of $S_{\mathrm{c}}$ and $\tau_{\mathrm{w}}$ values for benzene in USY, DAY and $\mathrm{NaY}$ in order to discuss the effects of the site-to-site hopping on $S_{\mathrm{c}}$ and the diffusion-related wobbling motion. A small characteristic order parameter $S_{\mathrm{c}}$ (or residual interaction $\omega_{\mathrm{c}}$ ) is found for a faujasite with more symmetrically arranged multi-adsorption sites in the same cage such as in NaY. The effects are also reflected in the DQF spectra recorded for benzene adsorbed in $\mathrm{NaX}$ frameworks. The spectra show less wiggling in the shoulder than for DAY and USY and thus one may obtain a small $S_{\mathrm{c}}$ value. $^{12}$ The magnitude of $P_{s}$ and $\tau_{\mathrm{i}}$ obtained in $\mathrm{NaX}$ are much smaller than those in $\mathrm{NaY}$. It is found that in $\mathrm{NaX}$ the $\tau_{\mathrm{i}}$ value is almost independent of loading. The $k_{f s}$ values are larger in $\mathrm{NaX}$ than in $\mathrm{NaY}$ owing to a larger diffusivity in the former system. Also, in $\mathrm{NaX}$ systems the correlation time $\tau_{\mathrm{w}}$ of the wobbling motion in low-loading samples is significantly smaller than that of high-loading samples. This may be due to the fact that the $\mathrm{S}_{\text {III }} \mathrm{Na}^{+}$in $\mathrm{NaX}$ may enhance the mutual hindrance of the molecules and hence reduce the benzene mobility. ${ }^{22}$ Nevertheless, negligible differences in $\tau_{\mathrm{w}}$ with variation of loading are found in $\mathrm{NaY}$, DAY and USY. In those systems, additional benzene molecules are less affected by the interaction with weak adsorption sites. Further, this effect is counterbalanced by the mutual hindrance of the benzene molecules in the same cage as loading increases. Hence, as listed in Table 5, the self-diffusion coefficients remain almost unchanged. The Arrhenius parameters of the obtained self-diffusion coefficients for different loadings and faujasite-type zeolites are the same as shown in Table 4 for wobbling motion. Moreover, it is worth noting that equlibrium and non-equilibrium methods, including quasi-elastic neutron scattering, ${ }^{23 \mathrm{a}}$ zero length column, ${ }^{23 \mathrm{~b}}$ piezometric ${ }^{23 \mathrm{c}}$ and gravimetric measurements, ${ }^{23 \mathrm{~d}}$ give diffusion coefficients of the order of $10^{-12}-10^{-9} \mathrm{~m}^{2} \mathrm{~s}^{-1}$ at $458 \mathrm{~K}$ for benzene adsorbed on $\mathrm{NaX}$. 

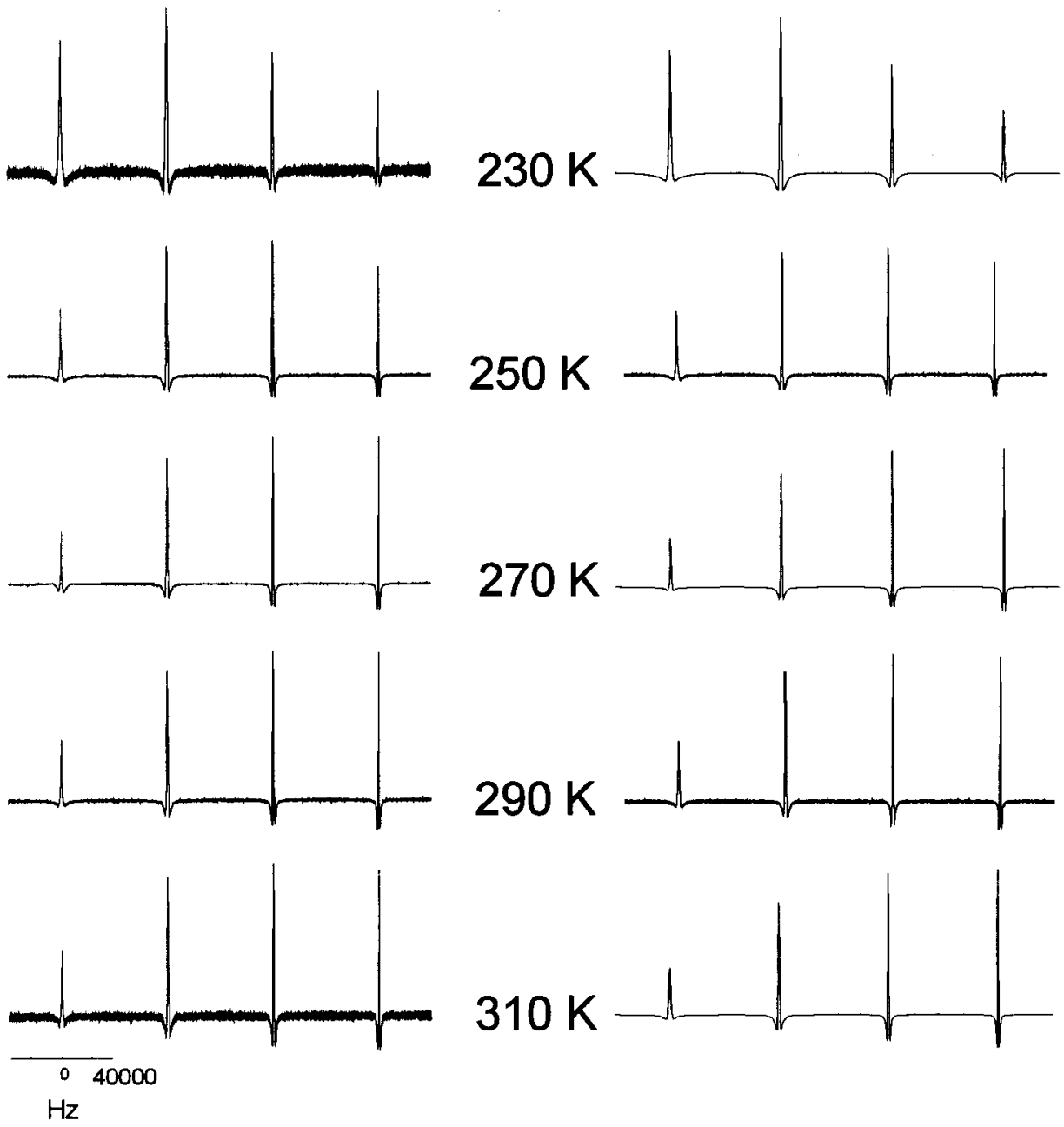

$\mathrm{Hz}$

Figure 1. Experimental (left) and simulated (right) ${ }^{2} \mathrm{H} D Q F$ spectra of the NaX low-loading sample at various temperatures. The four DQF spectra from left to right were obtained with DQ creation times of 40, 160, 280 and $400 \mu \mathrm{s}$, respectively. The spectral width is $\pm 40 \mathrm{kHz}$.

\section{DISCUSSION}

Auerbach et al. examined intracage and interacge benzene mobility in $\mathrm{NaX}(\mathrm{Si}: \mathrm{Al}=1.0)$ and $\mathrm{NaY}(\mathrm{Si}: \mathrm{Al}=2.0){ }^{4 \mathrm{f}}$ Their calculations indicate that the activation energy from PFG NMR is correlated with the site-to-window activation energies. Comparing the estimated self-diffusion coefficients in NaX with those from PFG NMR, the result yields satisfactory agreement. The Arrhenius analyses for self-diffusion coefficients from PFG NMR in NaX $(\mathrm{Si}: \mathrm{Al}=1.2)$ yield an activation energy of $21 \mathrm{~kJ} \mathrm{~mol}^{-1}$ at a loading between 1.8 and 3.8 and an activation energy of $28 \mathrm{~kJ} \mathrm{~mol}^{-1}$ at a loading of 4.9 and 5.3 , while present work yields $19 \mathrm{~kJ} \mathrm{~mol}^{-1}$ at 0.6 loading and $29 \mathrm{~kJ} \mathrm{~mol}^{-1}$ at 3.8 loading.

For benzene in DAY and USY, it is believed that the hydroxyl aluminum sites act as the adsorption sites. There are about 12 and 4.8 hydroxyl aluminum sites per unit cell of DAY and USY, respectively. However, one cannot be sure whether the locations of the hydroxyls are in the supercage or in the sodalite cage. Consequently, there are less than 1.5 and 0.6 hydroxl aluminum sites per supercage in DAY and USY, respectively. Owing to a small number of benzene sites and a weaker adsorption interaction in these systems, one may expect that the benzene possesses larger diffusivities with smaller activation energies in USY and DAY than in $\mathrm{NaY}$ and $\mathrm{NaX}$.

\section{Comparison with benzene orientation randomization scheme}

If the $S_{\text {II }}$ adsorption sites are perfectly tetrahedrally arranged and the benzene mobility is in the limit of fast site-to-site hopping, one may obtain full orientation randomization with a simple exponential decay. This is the case that DQF spectra may not be observed. Otherwise, as demonstrated by Auerbach and Metiu, ${ }^{4}$ the orientation randomization may yield a fast decay at the initial time due to the incomplete averaging in the site-to-site hopping processes. Then, in the time correlation function of the benzene orientation, it is followed by a much slower decay. ${ }^{4 \mathrm{e}}$ The decay rate of the latter process may be invoked in the calculation of the self-diffusion coefficient followed by $D=\frac{1}{6} k_{\mathrm{BOR}}\left\langle l^{2}\right\rangle$. The results agree well with the self-diffusion coefficients calculated from Eqn (6) with the measured $\tau_{\mathrm{w}}$ in $\mathrm{NaY}$ at a loading of 0.5 and 3.0, and the corresponding activation energies are 25 and $20 \mathrm{~kJ} \mathrm{~mol}^{-1}$, 

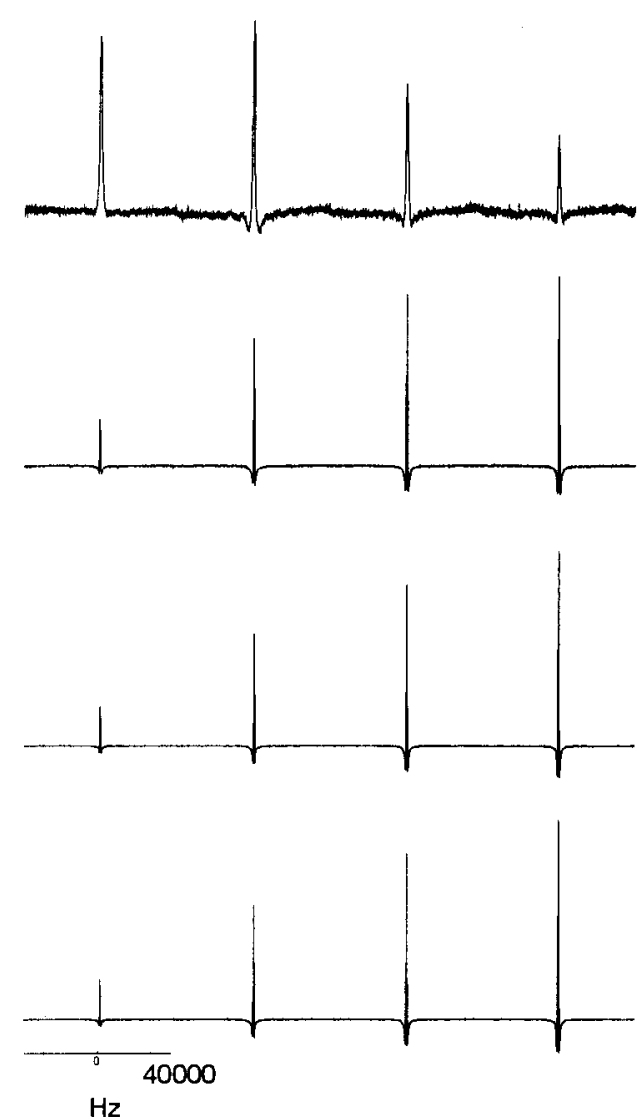
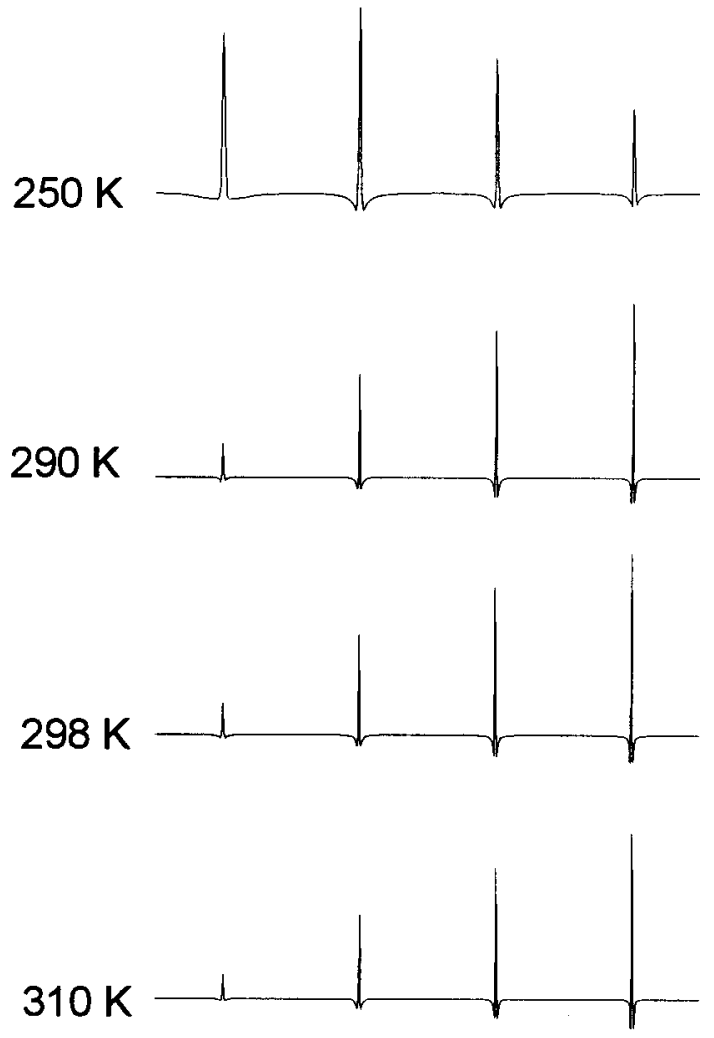

Figure 2. Experimental (left) and simulated (right) ${ }^{2} \mathrm{H}$ DQF spectra of the $\mathrm{NaX}$ high-loading sample at various temperatures. The four DQF spectra from left to right were obtained with DQ creation times of 40, 160, 280 and $400 \mu \mathrm{s}$, respectively. The spectral width is $\pm 40 \mathrm{kHz}$.

Table 1. The parameters used in the fitting of $T_{1}$, linewidth and the simulation of double quantum filtered spectra for NaX samples

\begin{tabular}{ccccccr}
\hline $\begin{array}{c}\text { Loading } \\
\text { (molecules/supercage) }\end{array}$ & $\begin{array}{c}\text { Temp. } \\
(\mathrm{K})\end{array}$ & $P_{s}^{\mathrm{a}}$ & $S_{c}^{\mathrm{a}}$ & $\begin{array}{c}\tau_{\mathrm{i}} \\
(\mathrm{ns})\end{array}$ & $\begin{array}{c}\tau_{\mathrm{w}} \\
(\mathrm{ns})\end{array}$ & $\begin{array}{r}k_{f s} \times 10^{-2} \\
\left(\mathrm{~s}^{-1}\right)^{\mathrm{a}}\end{array}$ \\
\hline 0.6 & 230 & 0.40 & 0.10 & $43 \pm 1$ & $300 \pm 10$ & 230 \\
& 250 & 0.33 & 0.14 & $32 \pm 1$ & $110 \pm 5$ & 206 \\
& 270 & 0.27 & 0.20 & $15 \pm 1$ & $50 \pm 3$ & 169 \\
& 290 & 0.25 & 0.25 & $12 \pm 1$ & $36 \pm 2$ & 208 \\
3.8 & 310 & 0.24 & 0.67 & $11 \pm 1$ & $24 \pm 2$ & 240 \\
& 250 & 0.35 & 0.2 & $32 \pm 3$ & $450 \pm 50$ & 140 \\
& 290 & 0.15 & 0.13 & $12 \pm 1$ & $70 \pm 30$ & 250 \\
& 298 & 0.14 & 0.13 & $9 \pm 1$ & $40 \pm 30$ & 290 \\
& 310 & 0.13 & 0.09 & $8 \pm 1$ & $33 \pm 30$ & 1900 \\
\hline
\end{tabular}

${ }^{\text {a }} 10 \%$ error is expected in $k_{f s}, 5 \%$ error in $P_{s}$ and $20 \%$ error in $S_{c}$.

respectively, compared with a value of $35 \mathrm{~kJ} \mathrm{~mol}^{-1}$ from $\mathrm{KMC}$ calculation. ${ }^{4 e}$ For benzene in $\mathrm{NaX}$, we predict an activation energy of $19 \mathrm{~kJ} \mathrm{~mol}^{-1}$ at 0.6 loading compared with an activation energy of $15 \mathrm{~kJ} \mathrm{~mol}^{-1}$ obtained from the BOR rate. ${ }^{4 \mathrm{f}}$

Following the KMC study, it is found that benzene adsorbed on the $\mathrm{NaX} \mathrm{S}_{\text {II }}$ site is by $7 \mathrm{~kJ} \mathrm{~mol}^{-1}$ less stable than on the $\mathrm{S}_{\text {II }}$ site in $\mathrm{NaY}$ but the adsorption strength on window sites remains unchanged..$^{4 f}$ Also, the study shows that $S_{\text {II }}$ and $S_{\text {III }}$ have similar binding energies with benzene. In Table 4 , the activation energy of diffusion in $\mathrm{NaX}$ obtained in present work appears to be $6 \mathrm{~kJ} \mathrm{~mol}^{-1}$ smaller than that in $\mathrm{NaY}$.

\section{Significance of previous $T_{1}$ and $T_{2}$ relaxation studies}

The enhancement of the benzene diffusivity from $\mathrm{NaY}$ to $\mathrm{NaX}$ is due to attractive interactions of $\mathrm{S}_{\text {III }}$ sites overlapping those from an adjacent $S_{\text {II }}$ site, making benzene mobility in $\mathrm{NaX}$ more energetically favorable. As pointed out by Auerbach et al., the agreement between NMR relaxation data and the PFG NMR measurements of benzene in $\mathrm{NaX}$ provides interesting insights. ${ }^{4 \mathrm{f}}$ It is supposed that only the intracage reorientational diffusion could account for the motional relaxation in the $T_{2}$ process. Furthermore, the additional $\mathrm{Na}^{+}$ions at $\mathrm{S}_{\mathrm{III}}$ sites affect both 
Table 2. Experimental and calculated ${ }^{2} \mathrm{H} T_{1}$ and linewidthin $\mathrm{NaX}$ samples measured at various temperatures and two resonance fields

\begin{tabular}{|c|c|c|c|c|c|c|c|c|c|}
\hline \multirow{3}{*}{$\begin{array}{c}\text { Loading } \\
\text { (molecules/supercage) }\end{array}$} & \multirow{3}{*}{$\underset{\substack{{ }^{\circ} \mathrm{C} \\
\text { Temperature }}}{ }$} & \multicolumn{4}{|c|}{$T_{1}(\mathrm{~ms})^{\mathrm{a}}$} & \multicolumn{4}{|c|}{$\Delta v_{1 / 2}(\mathrm{~Hz})^{\mathrm{a}}$} \\
\hline & & \multicolumn{2}{|c|}{$46.07 \mathrm{MHz}$} & \multicolumn{2}{|c|}{$76.78 \mathrm{MHz}$} & \multicolumn{2}{|c|}{$46.07 \mathrm{MHz}$} & \multicolumn{2}{|c|}{$76.78 \mathrm{MHz}$} \\
\hline & & Calc. & Exp. & Calc. & Exp. & Calc. & Exp. & Calc. & Exp. \\
\hline \multirow[t]{5}{*}{0.6} & 230 & 51 & 51 & 99 & 100 & 2300 & 2200 & 2100 & 2127 \\
\hline & 250 & 40 & 41 & 84 & 86 & 1551 & 1518 & 1540 & 1621 \\
\hline & 270 & 32 & 36 & 72 & 68 & 1176 & 1076 & 1294 & 1220 \\
\hline & 290 & 30 & 34 & 73 & 67 & 1000 & 1100 & 1152 & 1119 \\
\hline & 310 & 29 & 34 & 70 & 65 & 900 & 955 & 1020 & 1037 \\
\hline \multirow[t]{4}{*}{3.8} & 250 & 50 & 57 & 134 & 111 & 2210 & 2398 & 2560 & 2825 \\
\hline & 290 & 30 & 35 & 74 & 72 & 700 & 690 & 720 & 760 \\
\hline & 298 & 25 & 30 & 57 & 58 & 520 & 620 & 500 & 580 \\
\hline & 310 & 23 & 25 & 48 & 50 & 420 & 480 & 400 & 450 \\
\hline
\end{tabular}

${ }^{\mathrm{a}} \mathrm{A} 10 \%$ error is expected in all values.

Table 3. $S_{\mathrm{c}}$ and $\tau_{\mathrm{w}}$ parameters used in the fitting of $T_{1}$, linewidth and DQF spectra for faujasite samples ${ }^{11,12}$

\begin{tabular}{lcccc}
\hline $\begin{array}{l}\text { Zeolite } \\
\text { (Si : Al) }\end{array}$ & $\begin{array}{c}\text { Loading } \\
\text { (molecules/ } \\
\text { supercage) }\end{array}$ & $\begin{array}{c}\text { Temperature } \\
\left({ }^{\circ} \mathrm{C}\right)\end{array}$ & $S_{\mathrm{c}}^{\mathrm{a}}$ & $\begin{array}{c}\tau_{\mathrm{w}} \\
(\mathrm{ns})\end{array}$ \\
\hline $\mathrm{NaY}$ & 0.6 & 290 & 0.04 & $350 \pm 20$ \\
$(2.4)$ & & 310 & 0.02 & $220 \pm 30$ \\
& & 330 & 0.02 & $120 \pm 10$ \\
& & 350 & 0.02 & $60 \pm 5$ \\
& 3.0 & 290 & 0.04 & $300 \pm 20$ \\
& & 310 & 0.02 & $210 \pm 25$ \\
DAY & 0.8 & 330 & 0.02 & $110 \pm 15$ \\
$(15)$ & & 210 & 0.9 & $50 \pm 4$ \\
& & 250 & 0.5 & $20 \pm 2$ \\
& & 290 & 0.3 & $13 \pm 1$ \\
& 3.9 & 330 & 0.3 & $10 \pm 1$ \\
& & 210 & 0.9 & $10 \pm 2$ \\
USY & & 250 & 0.3 & $10 \pm 3$ \\
$(40)$ & 0.5 & 290 & 0.3 & $10 \pm 3$ \\
& & 210 & 1.1 & $70 \pm 5$ \\
& & 250 & 1.4 & $16 \pm 2$ \\
& & 290 & 1.7 & $7.5 \pm 1$ \\
& 3.2 & 330 & 5.0 & $4.5 \pm 3$ \\
& & 210 & 0.2 & $40 \pm 5$ \\
& & 250 & 0.3 & $14 \pm 2$ \\
& & 290 & 0.3 & $8 \pm 3$ \\
\hline A & & & &
\end{tabular}

${ }^{\text {a }}$ A $20 \%$ error is expected in $S_{\mathrm{c}}$ values.

intercage and intracage hopping activation energies and make them approximately equal. This explains why the diffusion coefficients obtained from NMR relaxation data agree remarkably well with those from PFG NMR for benzene in $\mathrm{NaX}$. Furthermore, the results from the KMC studies in $\mathrm{NaY}$ suggest that estimates of the diffusion from the reorientational correlation time deduced from $T_{1}$ and $T_{2}$ studies is inappropriate since its time-scale is believed to be correlated with the orientational randomization. The self-diffusion coefficient obtained should not agree with the PFG NMR results. However, the time-scale from $T_{2}$ fitting in $\mathrm{NaY}$ is of the order of $10^{-7} \mathrm{~s}$, which is about one order of magnitude slower than the site-to-site hopping
Table 4. Activation energy $E_{\mathrm{a}}$ and Arrhenius prefacters $A$ of the wobbling motion for benzene in NaY, DAY, USY and $\mathrm{NaX}$ samples

\begin{tabular}{|c|c|c|c|c|}
\hline Zeolite & $\begin{array}{c}\text { Loading } \\
\text { (molecules/ } \\
\text { supercage) }\end{array}$ & $\begin{array}{c}A \\
\left(\mathrm{~s}^{-1}\right)\end{array}$ & $\begin{array}{c}E_{\mathrm{a}} \\
\left(\mathrm{kJ} \mathrm{mol}^{-1}\right)\end{array}$ & $\begin{array}{c}\text { Temperature } \\
\text { range }(\mathrm{K})\end{array}$ \\
\hline \multirow[t]{2}{*}{$\mathrm{NaY}$} & 0.5 & $7.4 \pm 0.3 \times 10^{10}$ & $25 \pm 2$ & $290-350$ \\
\hline & 3.0 & $1.2 \pm 0.6 \times 10^{10}$ & $20 \pm 4$ & $290-330$ \\
\hline DAY & 0.8 & $1.8 \pm 0.1 \times 10^{9}$ & $8 \pm 0.8$ & $210-330$ \\
\hline \multirow[t]{2}{*}{ USY } & 0.5 & $6.8 \pm 0.1 \times 10^{10}$ & $15 \pm 0.7$ & $210-330$ \\
\hline & 3.2 & $9.3 \pm 0.2 \times 10^{9}$ & $10 \pm 0.8$ & $210-290$ \\
\hline \multirow[t]{2}{*}{$\mathrm{NaX}$} & 0.6 & $7.1 \pm 0.2 \times 10^{10}$ & $19 \pm 2$ & $210-310$ \\
\hline & 3.8 & $2.5 \pm 0.1 \times 10^{12}$ & $29 \pm 2$ & $210-310$ \\
\hline
\end{tabular}

rate. Therefore, the agreement in some way reflects the slower mode of motional process. As shown in Table 5, the self-diffusion coefficients of benzene estimated from $T_{2}$ measurements in $\mathrm{NaY}$ and $\mathrm{NaX}$ agree quantitatively with our result. ${ }^{7,19}$ The motional correlation time obtained from $T_{2}$ relaxation studies to estimate the self-diffusion coefficient may be correlated with the $\tau_{\mathrm{w}}$ process of wobbling motion in terms of the present model with a small $S_{\mathrm{c}}$ value. However, the self-diffusion coefficient of benzene estimated from $T_{1}$ measurement in NaY and ZDDAY ${ }^{24}$ may reflect the effects from the fast motional mode such as the site-to-site hopping or/and the internal rotation rather than from the slow mode of the wobbling motion. The estimated self-diffusion coefficient is about one order of magnitude larger than the value estimated from $T_{2}$ studies. ${ }^{7,19}$

Owing to a small number of benzene sites and a weaker adsorption interaction in USY and DAY, one may expect a small $\tau_{\mathrm{w}}$ in USY and DAY. Particularly in USY, there is lack of sites for intracage hopping. It is obvious that the significance of the $\tau_{\mathrm{w}}$ process is related to the site-to-cage hopping only. An analogous argument is also valid for the ZDDAY system. Therefore, the correlation time calculated from $T_{1}$ accounts for the process of internal rotation only and, consequently, the self-diffusion coefficient obtained loses its physical ground. 
Table 5. Self-diffusion coefficient $(D)$ obtained from DQF spectral analysis and other methods at $298 \mathrm{~K}$

\begin{tabular}{|c|c|c|c|}
\hline $\begin{array}{l}\text { Zeolite } \\
(\mathrm{Si}: \mathrm{Al})\end{array}$ & $\begin{array}{c}\text { Loading } \\
\text { (molecules/ } \\
\text { supercage) }\end{array}$ & $\begin{array}{c}D \\
\left(\mathrm{~m}^{2} \mathrm{~s}^{-1}\right)\end{array}$ & Method \\
\hline $\mathrm{NaX}(1.2)$ & 1.1 & $1 \times 10^{-11}$ & $\mathrm{PFG}^{23 \mathrm{~d}}$ \\
\hline $\mathrm{NaX}(1.2)$ & 1.8 & $3 \times 10^{-11}$ & $\mathrm{PFG}^{22}$ \\
\hline $\mathrm{NaX}(1.2)$ & 1 & $1 \times 10^{-11}$ & $\mathrm{~T}_{2}^{18}$ \\
\hline $\operatorname{NaX}(1.2)$ & 3.6 & $9 \times 10^{-12}$ & $\mathrm{PFG}^{23 \mathrm{~d}}$ \\
\hline $\operatorname{NaX}(1.2)^{\mathrm{a}}$ & 3.8 & $1 \times 10^{-11}$ & $\mathrm{PFG}^{22}$ \\
\hline $\mathrm{NaX}(1.2)$ & 3.8 & $5 \times 10^{-12}$ & DQF \\
\hline $\mathrm{NaX}(1.2)$ & 0.6 & $7 \times 10^{-12}$ & $\mathrm{DQF}$ \\
\hline $\mathrm{NaY}(2.7)$ & 1 & $8 \times 10^{-13}$ & $\mathrm{~T}_{2}^{7}$ \\
\hline $\operatorname{NaY}(2.7)$ & 1 & $1 \times 10^{-13}$ & $\mathrm{~T}_{2}^{7}$ \\
\hline $\mathrm{NaY}(1.7)$ & 1 & $4 \times 10^{-12}$ & $\mathrm{~T}_{1}^{24}$ \\
\hline $\mathrm{NaY}$ (3) & & $5 \times 10^{-13}$ & $\mathrm{KMC}^{4 \mathrm{e}}$ \\
\hline $\mathrm{NaY}(2.4)$ & 0.5 & $7 \times 10^{-13}$ & DQF \\
\hline $\mathrm{NaY}(2.4)$ & 3.0 & $8 \times 10^{-13}$ & $\mathrm{DQF}$ \\
\hline DAY (15) & 0.8 & $2 \times 10^{-11}$ & $\mathrm{DQF}$ \\
\hline $\operatorname{DAY}(15)^{\mathrm{a}}$ & 3.9 & $2 \times 10^{-11}$ & $\mathrm{DQF}$ \\
\hline USY (40) & 0.9 & $2 \times 10^{-11}$ & $\mathrm{~T}_{2}^{7}$ \\
\hline USY (40) & 0.9 & $1 \times 10^{-12}$ & $\mathrm{~T}_{2}^{7}$ \\
\hline ZDDAY & 1 & $5 \times 10^{-10}$ & $\mathrm{~T}_{1}^{24}$ \\
\hline USY (40) & 0.5 & $3 \times 10^{-11}$ & DQF \\
\hline $\operatorname{USY}(40)^{\mathrm{a}}$ & 3.2 & $3 \times 10^{-11}$ & DQF \\
\hline
\end{tabular}

${ }^{\mathrm{a}}$ Extrapolated value.

\section{Comparison with ${ }^{2} \mathrm{H}$ NMR exchange experiments}

Isfort et al. used ${ }^{2} \mathrm{H}$ NMR exchange experiments to study the reorientational dynamics of benzene molecules at full occupancy of the supercages of NaY. ${ }^{9}$ Experimental 2D echo time-domain decay profiles were obtained. Comparison with model calculation gives evidence for tetrahedral-like jumps associated with the small-angle 'reorientation' motion for benzene molecules in the neighborhood. The small-angle reorientation of $\mathrm{C}_{6} \mathrm{D}_{6}$ is interpreted to be due to instantaneous relaxations after the hopping of neighboring molecules in the supercages. Although both the wobbling motion described here and the small-angle reorientation found by Isfort et al. result in small-angle reorientation for benzene molecules on adsorption sites, the former is related to $S_{I I}-W$ hopping whereas the latter is associated with $\mathrm{S}_{\mathrm{II}}-\mathrm{S}_{\mathrm{II}}$ hopping. Since there are differences in the time-scales and the physical origins of the above two processes, one may decouple these two processes. Even without intracage tetrahedral jumps between adjacent sites, e.g. in the USY samples, the wobbling motion still exists and it is associated with intercage diffusion. However, in the case of benzene adsorbed on $\mathrm{CaX},{ }^{13} \mathrm{C}$ exchange-induced sideband (EIS) NMR indicates the very slow diffusion process which yields self-diffusion coefficients of the order of $10^{-18}-10^{-19} \mathrm{~m}^{2} \mathrm{~s}^{-1}$ over the temperature range $338-368 \mathrm{~K}^{10}$ The existence of a slower motional mode may account for the discrepancy in the diffusion coefficients observed.

\section{CONCLUSION}

We have presented a more complete relaxation study to ensure the evaluation of the correlation time of wobbling motion in order to estimate the self-diffusion coefficient of benzene in faujastie-type zeolites. Comparisons with previous $T_{1}, T_{2}$ and PFG NMR measurements were made. Discussions of the validity of estimation from such studies were also given in terms of the motional modes involved in spin relaxations. DQF spectral analysis in conjunction with the usual relaxation studies may be a better combination of NMR methods for characterizing the dynamics in the zeolite system. This study forms part of a continuing series of studies of NMR methods for the investigation of diffusion and adsorption in porous materials.

\section{Acknowledgment}

Financial support from the National Science Council of the Republic of China under Grant No. NSC 88-2113-M-002-00 to this project is gratefully acknowledged.

\section{REFERENCES}

1. H. Lechert, Catal. Rev. Sci. Eng. 14, 1 (1976).

2. R. Eckmana and A. J. Vega, J. Am. Chem. Soc. 105, 4841 (1983).

3. D. Barthomeuf, Catal. Rev. 38, 521 (1996).

4. (a) C. Saravanan and S. M. Auerbach, J. Chem. Phys. 107, 8120 (1997). (b) C. Saravanan and S. M. Auerbach, J. Chem. Phys. 107, 8132 (1997). (c) C. Saravanan, J. Fabien and S. M. Auerbach, J. Chem. Phys. 108, 2162 (1998). (d) S. M. Auerbach, J. Chem. Phys. 106, 7810 (1997). (e) S. M. Auerbach and H. I. Metiu, J. Chem. Phys. 106, 2893 (1997). (f) S. M. Auerbach, L. M. Bull, N. J. Henson and H. I. Metiu, J. Phys. Chem. 100, 5923 (1996). (g) S. M. Auerbach and H. I. Metiu, J. Chem. Phys. 105, 3753 (1996). (h) S. M. Auerbach, N. J. Henson, A. K. Cheetham and H. I. Metiu, J. Phys. Chem. 99, 10600 (1995).

5. (a) B. Zibrowius, J. Caro and H. Pfeifer, J. Chem. Soc. Faraday Trans. 1 84, 2347 (1988). (b) H. Pfeifer, in NMR-Basic Principles and Progress; Springer, Berlin, Vol. 7, p. 53 (1972). (c) H. Pfeifer, Phys. Reports (Section C of Physics Letters) 26, 293 (1976).

6. G. Vitale, L. M. Bull, R. E. Morris, A. K. Cheetham, B. H. Toby, C. G. Coe and J. E. MacDougall, J. Phys. Chem. 99, 16087 (1995).

7. J. A. Sousa Goncalves, R. L. Portsmouth, P. Alexander and L. F. Gladden, J. Phys. Chem. 99, 3317 (1995).

8. D. J. Schaefer, D. E. Favre, M. Wilhelm, S. J. Weigel and B. F. Chmelka, J. Am. Chem. Soc. 119, 9252 (1997).

9. O. Isfort, B. Boddenberg, F. Fujara and R. Grosse, Chem. Phys. Lett. 288, 71 (1998)

10. D. E. Favre, D. J. Schaefer, S. M. Auerbach and B. F. Chmelka, Phys. Rev. Lett. 81, 5852 (1998).

11. Y.-H. Chen, W.-T. Chang, P.-C. Jiang and L.-P. Hwang, Microporous Mesoporous Mater. 21, 651 (1998).

12. Y.-H. Chen and L.-P. Hwang, J. Phys. Chem. B 103, 5070 (1999).

13. J. R. Brainard and A. Szabo, Biochemistry 20, 4618 (1981).

14. R. R. Ernst, G. Bodenhausen and A. Wokan, in Principles of Nuclear Magnetic Resonance in One and Two Dimensions, p. 49 Clarendon, Oxford (1987).

15. D. M. Brink and G. R. Satchler, in Angular Momentum, p. 28 London, Oxford Univ. Press (1968).

16. F. S. Millett, B.P. Dailey, J. Chem. Phys. 56, 3249 (1972).

17. W. S. Price, N.-H. Ge, L.-Z. Hong and L.-P. Hwang, J. Am. Chem. Soc. 115, 1095 (1993).

18. J. P. Jacobsen, H. K. Bildsoe and K. Schaumburg, J. Magn. Reson. 23, 153 (1976).

19. B. Boddenberg and R. Burmeister, Zeolite 8, 488 (1988).

20. T. C. Wong, P.-L. Wang, D.-M. Duh and L.-P. Hwang, J. Phys. Chem. 93, 1295 (1989).

21. Y. Sharf, U. Eliav, H. Shinar and G. Navon, J. Magn. Reson. B107, 60 (1995).

22. A. Germanus, J. Kärger, H. Pfeifer, N. N. Samulevic and S. P. Zdanov, Zeolites 5, 91 (1985). 
23. (a) H. Jobic, M. Bée, J. Kärger, H. Pfeifer and J. Caro, J. Chem. Soc., Chem. Commun. 341 (1990). (b) M. Eic, N. V. Goddard and D. M. Ruthven, Zeolites 8, 327 (1988). (c) M. Bülow, W. Meith, P. Struve and P. Lorenz, J. Chem. Soc. Faraday Trans. 1 79, 2457
(1983). (d) J. Kärger and D. M. Ruthven, J. Chem. Soc. Faraday Trans. 1 77, 1485 (1981).

24. L. M. Bull, N. J. Henson, A. K. Cheetham, J. M. Newsam and S. J. Heyes, J. Phys. Chem. 97, 11776 (1993). 